\title{
Role of Long-Acting Injectable Second-Generation Antipsychotics in the Treatment of First-Episode Schizophrenia: A Clinical Perspective
}

\author{
Radovan Přikryl, ${ }^{1,2}$ Hana Přikrylová Kučerová, ${ }^{1,2}$ Michaela Vrzalová, ${ }^{2}$ and Eva Češková ${ }^{1,2}$ \\ ${ }^{1}$ Central European Institute of Technology, Masaryk University (CEITEC-MU), Kamenice 753/5, 62500 Brno, Czech Republic \\ ${ }^{2}$ Department of Psychiatry, Faculty of Medicine Masaryk University, University Hospital Brno, \\ Jihlavska 20, 62500 Brno, Czech Republic
}

Correspondence should be addressed to Radovan Přikryl, radovan.prikryl@post.cz

Received 24 October 2011; Revised 15 February 2012; Accepted 15 February 2012

Academic Editor: Dawn I. Velligan

Copyright (C 2012 Radovan Prikryl et al. This is an open access article distributed under the Creative Commons Attribution License, which permits unrestricted use, distribution, and reproduction in any medium, provided the original work is properly cited.

\begin{abstract}
Approximately $80 \%$ of patients with the first-episode schizophrenia reach symptomatic remission after antipsychotic therapy. However, within two years most of them relapse, mainly due to low levels of insight into the illness and nonadherence to their oral medication. Therefore, although the formal data available is limited, many experts recommend prescribing long-acting injectable second-generation antipsychotics (mostly risperidone or alternatively paliperidone) in the early stages of schizophrenia, particularly in patients who have benefited from the original oral molecule in the past and agree to receive long-term injectable treatment. Early application of long-acting injectable second-generation antipsychotics can significantly reduce the risk of relapse in the future and thus improve not only the social and working potential of patients with schizophrenia but also their quality of life.
\end{abstract}

\section{Depot Antipsychotics for the Treatment of Schizophrenia}

First-generation long-acting injectable (depot) antipsychotics (AP1G) emerged in clinical practice in the 1960s. For the treatment of schizophrenia, their use resulted in a significant decrease in the number of patients relapses, including length and frequency of hospitalizations [1]. However, when oral second-generation antipsychotics (AP2G) were introduced thirty years later, the position (in clinical practice) of depot AP1G dramatically changed. Despite the benefits of AP1G, psychiatrists prescribed them for longterm therapy of schizophrenia much less often and started to switch to oral AP2G as they were seen as more efficient and better tolerated [2]. This trend persisted for many years, despite evidence to suggest from meta-analyses and naturalistic studies that depot AP1G were more effective in reducing schizophrenic relapses than oral AP2G [3]. The same finding was later logically replicated also for long-acting injectable (LAI) AP2G [4-7]. Although patients with schizophrenia are often willing to use depot or LAI antipsychotics, these preparations are today prescribed only for approximately $20 \%$ of them [8-10]. In a survey [11] psychiatrists answered that they only offer long-acting injectable antipsychotics to one in every three patients with schizophrenia.

\section{Specifics for First-Episode Schizophrenia Therapy}

Therapy for the first episode of schizophrenia has certain specific features. Patients respond to low doses of antipsychotics relatively well; yet they are more sensitive to the adverse effects, particularly extrapyramidal ones $[12,13]$. Patients 
with first-episode schizophrenia, however, typically show low willingness to use antipsychotics in the long-term treatment, likely due to their high unawareness of the disease severity. Recommended duration of antipsychotic administration after the first episode of schizophrenia usually ranges from 1 to 2 years [12-14]. A five-year observation study of firstepisode patients showed that the risk of relapse is five times higher after discontinuation of therapy compared to continuous medication [15]. Despite the recommendations from their psychiatrists, patients with schizophrenia discontinue their therapy often. Results of the clinical antipsychotic trials of intervention effectiveness (CATIE) study indicate that up to $74 \%$ of patients with schizophrenia discontinued their therapy after 18 months; the European First Episode Schizophrenia Trial (EUFEST) shows that up to $42 \%$ of patients terminated their treatment within one year after the disease onset $[16,17]$. When considering the length of subsequent prophylactic treatment after the first episode of schizophrenia; not only its efficacy but also the profile or severity of adverse events of the given antipsychotic must be taken into account. Moreover, it should be taken into consideration that approximately $20 \%$ of first-episode patients will never experience a subsequent exacerbation of schizophrenia, irrespective of whether they receive or the type of therapy they receive $[14,18]$.

\section{Problems Associated with Nonadherence to Pharmacological Treatment of Schizophrenia}

Schizophrenia is a chronic mental disease characterized among other things by a high degree of nonadherence to prescribed medication [19]. It has been reported that patients take on average only $58 \%$ of their prescribed drugs, $41.2 \%$ of patients do not use their medication according to prescription, and one- to two-thirds of patients take their pills irregularly [20-22]. These are the results of questionnaire surveys, and therefore they reflect only the situation of patients that agreed to take part in the research; in reality, therefore the rates of nonadherence may be much higher. In general, the current guidelines on schizophrenia treatment consider depot or long-acting injectable antipsychotics as drugs of choice for long-term therapy in patients who are nonadherent with antipsychotic medication [12-14]. Systematic surveys of specific studies indicate that patients treated with depot antipsychotics show only a $24 \%$ non-adherence rate while they are sufficiently covered by medication for $91 \%$ of the total therapy time [23-25].

\section{Long-Acting Injectable versus Oral Antipsychotics in the Treatment of Schizophrenia}

Since available studies differ in methodology and primary observation goals [26], it is difficult to generalize on the effects of oral or long-acting injectable administration of antipsychotics on the course of schizophrenia. In a metaanalysis, Adams et al. [27] did not prove dominance of depot AP1G over oral antipsychotics in terms of reduction of the number of relapses. Nevertheless, overall improvement was seen more often in patients treated with depot antipsychotics [27]. On the other hand, a recent meta-analysis [7] found lower levels of relapse in patients with schizophrenia treated with depot AP1G or LAI AP2G, compared with those treated with oral antipsychotics. Still, the two therapeutic strategies did not differ in the number of rehospitalizations, terminations of therapy, or cases of non-adherence. Inconsistency of results might be explained by the nature of double-blind randomized studies that, of course, do not typically enroll non-adherent patients who are likely to benefit most from the depot/LAI formulation of antipsychotic therapy. Conditions of real clinical practice are therefore better simulated by observation studies where depot and oral antipsychotics are often compared with a mirror design. These studies describe almost consistently lower number of days of hospitalization during depot or LAI antipsychotic therapy, as compared with the same period of oral medication $[1,28]$. The main methodological problem of these studies lies in the fact that, when the initial oral antipsychotic medication fails, a new oral treatment is prescribed, whilst the injectable medication remains the same. This can significantly affect the comparison of the therapy efficacy in identical time periods [26].

\section{Long-Acting Injectable Antipsychotics in First-Episode Schizophrenia}

Depot AP1Gs, let alone LAI AP2G, are rarely prescribed for patients with first-episode schizophrenia, although their levels of non-adherence and hence the risk of subsequent relapse are in reality very high $[29,30]$. Importantly, adverse course of early schizophrenia has a profound negative influence on psychosocial integration of patients, not to mention a patients ability to remain in education and/or employment. Economic consequences of schizophrenia therefore represent a burden not only for health system but also for the social system of public health insurance. Psychiatrists usually explain the low prescription rate of LAI antipsychotics in the initial/early stage of schizophrenia treatment as an unwillingness of patient to receive injections on an outpatient basis or generally by negative attitudes to depot/LAI antipsychotics $[31,32]$.

\section{Long-Acting Injectable Antipsychotics in First-Episode Schizophrenia: Attitudes of Psychiatrists}

Heres et al. [33] used a questionnaire to ask almost 200 German psychiatrists at the national congress in 2008 about their attitudes towards the use of LAI antipsychotics in patients with first-episode schizophrenia. They found that long-term injectable therapy was only offered to $26.7 \%$ of patients with first-episode schizophrenia; it was actually prescribed to $13.3 \%$ of those offered (i.e., one of every 
two). Respondents also reported that up to $60.4 \%$ patients taking antipsychotics irregularly will relapse within one year after the first episode [33]. The main outcome of this questionnaire was that it identified the three principal reasons for not using LAI antipsychotics in patients with first-episode schizophrenia. German psychiatrists stated that they find it difficult to present the benefits of a long-term injectable antipsychotic therapy to patients who do not have any personal experience with schizophrenia relapse, as they are still at the very beginning of their illness. Poor availability of LAI AP2G was identified as another reason, particularly when AP2G are strongly preferred over AP1G, especially in patients with first-episode schizophrenia. The last reason was mainly personal reservations of the psychiatrists associated associated with difficult control of adverse effects of depot antipsychotics, negative impact on patient-psychiatrist relationship, or higher amount of psychiatrist's time needed to manage depot medication administration [33]. The survey indicates that the only barrier to more frequent use of LAI AP2G in patients with first-episode schizophrenia, which is not influenced by perceptions of patients and psychiatrists, is the issue with market availability, price, and method of reimbursement. At present, only LAI risperidone, olanzapine pamoate, and paliperidone palmitate are available in most countries. For many reasons, limited availability of these drugs is today considered as the main barrier of their more common use in the therapy of schizophrenia [2, 11, 32]. This is problem mainly in first-episode patients in whom AP2G are strongly preferred as drugs of first choice [13, $34,35]$. Hopefully, better availability of LAI AP2G, simpler prescription rules, and better reimbursement from public health insurance will result in more common use of these products in clinical practice. However, this logical sequence of thoughts is in conflict with the real situation in the UK where better availability of LAI risperidone did not increase the use of LAI antipsychotics for treatment of schizophrenia despite previous belief of British psychiatrists that availability of these products was highly desirable and would significantly change their prescription habits in favor of LAI antipsychotics $[2,32]$. Keeping this in mind, the latter assumption that better availability of LAI AP2G would make psychiatrists prescribe LAI preparations more often sounds ironic [32]. Other reasons for not prescribing LAIs reflect subjective attitudes of psychiatrists and patients towards injectable therapy in general. The questionnaire by Heres et al. clearly highlighted that only one in four patients with first-episode schizophrenia was offered the possibility of treatment with LAI AP2G and half of those that were, agreed. That means that three out of four patients were not offered this type of therapy from their psychiatrist. Actually, the fact that psychiatrists, driven by their own personal negative attitudes/perceptions, offer LAI AP2G less frequently is a true barrier to using these products more commonly in patients with first-episode schizophrenia. Psychiatrists often say that first-episode patients reject LAI AP2G because they have not yet experienced a schizophrenic relapse. In this sense, higher efficacy of a LAI AP2G in prevention of relapses may not be a feasible argument $[1,5,36]$. Alternatively, low prescription rates can be explained by the fact that psychiatrists suppose beforehand (without discussing the issue with patients) that first-episode patients will not be interested in LAI AP2G therapy for the above-mentioned reasons. Interestingly, high self-confidence of psychiatrists to foreknow attitudes of their patients is considered to be one of the key factors of less frequent use of LAI AP2G in clinical practice $[2,32]$.

\section{Long-Acting Injectable Antipsychotics in First-Episode Schizophrenia: Current Knowledge}

Recent studies show that long-term injectable AP2G therapy is effective and also acceptable for first-episode patients, which is in opposition to the above-mentioned "conservative" attitudes [30, 37]. Unfortunately, studies comparing long-term injection or depot therapy with oral antipsychotic treatment after the first episode of schizophrenia are rare [38]. From this perspective, unique data can be drawn from a nation wide cohort study aimed at identification of the risk of rehospitalization and therapy discontinuation in more than 2,500 patients first hospitalized for schizophrenia between 2000 and 2007 in Finland [26]. The results show that in Finland, where antipsychotics are fully reimbursed from general health insurance systems, only $45.7 \%$ patients pick up prescribed drugs within one month and continue their therapy. Data are highly representative, since the study used the national registry and incorporated every single patient in Finland who was hospitalized for schizophrenia for the first time. Although information about medication during hospitalization was not available, almost all patients were recommended to start subsequent antipsychotic therapy. As compared with studies such as CATIE [16] or EUFEST [17], the Finnish study indicated higher rate of therapy discontinuation. This could be explained by the nature of the study which made it possible to capture real everyday behavior of patients, irrespective of their motivations or willingness to cooperate. As Finland, just as the majority of other countries, has not established any compulsory outpatient psychiatric screening, comparison of efficiency of individual antipsychotics can be generalized only for persons who were willing to visit their psychiatrist on an outpatient basis. It turned out that administration of depot AP1G and LAI risperidone resulted in reduction of the risk of rehospitalization by $50 \%$ or $65 \%$, respectively, compared with oral formulations of the same antipsychotics. Depot AP1G or LAI risperidone were the first-choice drug in $8 \%$ of patients, and, in total, they comprised $10 \%$ of treated patientyears [26]. This is relatively low, especially when compared with patients with chronic schizophrenia. So far, these antipsychotic formulations have been earmarked mainly for patients with low insight into the illness and poor adherence to the therapy. However, if the target population for LAI AP2G would be extended to include also patients with better insight and apparent adherence, rehospitalization rate should decrease. Of course, this would apply only to patients who are willing to use these products on an outpatient basis. 
TABLE 1: Survey of studies on LAI risperidone in first-episode schizophrenia patients.

\begin{tabular}{|c|c|c|c|c|c|}
\hline Author, year & Study design & $\begin{array}{l}\text { Duration of } \\
\text { schizophrenia }\end{array}$ & $N$ & Comparator & Result \\
\hline $\begin{array}{l}\text { Parellada et al., } \\
2005 \text { [39] }\end{array}$ & $\begin{array}{l}\text { Six-month } \\
\text { open study }\end{array}$ & $\begin{array}{l}\text { Median one year, no } \\
\text { more than } 3 \text { years }\end{array}$ & 382 & 0 & $\begin{array}{l}73 \% \text { patients completed the study, statistically significant } \\
\text { decrease of PANSS; } 40 \% \text { patients achieved at least } 20 \% \\
\text { reduction of total PANSS score. }\end{array}$ \\
\hline $\begin{array}{l}\text { Malla et al., } \\
2006[44]\end{array}$ & $\begin{array}{l}\text { Two-year } \\
\text { open study }\end{array}$ & Up to 3 years & 15 & Oral AP2G & $\begin{array}{l}\text { More significant reduction of total PANSS score versus } \\
\text { oral risperidone. }\end{array}$ \\
\hline $\begin{array}{l}\text { Emsley et al. } \\
2009[30]\end{array}$ & $\begin{array}{l}\text { Two-year } \\
\text { open study }\end{array}$ & First episode & 50 & 0 & $\begin{array}{l}72 \% \text { patients completed the study; } 78 \% \text { of them reached } \\
\text { at least } 50 \% \text { reduction of symptoms; remission persisted } \\
\text { for two years in } 62 \% \text { patients [ } 42] \text {. }\end{array}$ \\
\hline
\end{tabular}

\section{Long-Acting Injectable Second-Generation Antipsychotics in First-Episode Schizophrenia}

As far as LAI AP2G products are concerned, researchers have clinical experience with LAI risperidone, olanzapine pamoate, and paliperidone palmitate. Although many studies in schizophrenia with these products have been performed, relatively limited data on their use specifically in first-episode patients is available. Only LAI risperidone has been specifically studied for the use in early stage of schizophrenia (see Table 1) in addition specific posthoc analyses have been performed in patients classified as having recent onset schizophrenia. Parellada et al. [39] designed a six-month open-label study with LAI risperidone to analyze a subgroup of 382 patients with recent schizophrenia or schizoaffective disorder (diagnosed $\leq 3$ years ago) [40]. Schizophrenia was diagnosed in $84 \%$ of patients, with median of one year after the diagnosis was established. Previous medication included mainly AP2G (70\%) and depot AP1G (24\%). Non-adherence (42\%) and poor efficacy $(31 \%)$ of previous medication were the main reasons for changing the therapy. The study was completed by $73 \%$ patients who showed significant decrease of severity of schizophrenic symptomatology, reflected by statistically significant reduction of not only total PANSS score (positive and negative syndrom scale) [41] but also all PANSS subscales. In $40 \%$ of patients, total PANSS score decreased by at least $20 \%$. At the same time, patients showed an improvement of overall functioning, quality of life, and satisfaction [39]. In other study, Emsley et al. [30] administered LAI risperidone monotherapy to fifty patients with the first episode of schizophrenia: this two-year observation study was completed by 36 patients $(72 \%), 39$ patients $(78 \%)$ showed reduction of symptoms by at least $50 \%$; 4 of them relapsed, 32 patients (64\%) reached remission according to the proposed criteria for the remission in schizophrenia [42], and 31 patients $(62 \%)$ reached remission that persisted throughout the two years of the study [30]. After two years, 33 patients decided to terminate the therapy. Seventy-nine percent $(79 \%)$ of those then subsequently relapsed (median to relapse: 163 days). Based on these results, Emsley et al. [43] assert that first-episode patients who reached remission are prone to relapse after discontinuation of continuous long-acting injectable risperidone therapy. Antipsychotic treatment in such patients should therefore be continuous and maintained for at least two years [43]. Malla et al. [44] published data from their two-year prospective open multicentric study that was performed with young patients (aged 18 to 30 years) suffering from schizophreniform disorder, schizophrenia, or schizoaffective disorder (for no longer than 3 years). Patients were randomized to treatment with oral AP2G or LAI risperidone. Although it is difficult to generalize the results due to low number of subjects enrolled in the study $(n=15)$, patients treated with LAI risperidone showed more significant reduction of total PANSS score (by 16.1), as compared with oral AP2G (by 5.0) [44]. Weiden et al. [37] published preliminary data about early adherence from their randomized controlled study that compared LAI risperidone with oral AP2G in first-episode patients [37]. Nineteen $(19,73 \%)$ of 26 patients who were asked to participate in the study agreed to be treated with LAI risperidone; the other group consisted of 11 patients. Adherence rate in the twelfth week of observation was comparable in both groups. However, adherence questionnaires indicated that patients accepting LAI risperidone therapy showed higher probability of adherence, as compared with patients treated with oral antipsychotics [37]. Bartzokis et al. [45] examined the impact of antipsychotic formulation on the myelination trajectory during a randomized six-month trial of LAI risperidone versus oral risperidone in first-episode schizophrenia subjects. Two groups (11 patients treated with LAI risperidone and 13 patients treated with oral risperidone) that were matched in prerandomization oral medication exposure and 14 healthy controls were prospectively examined. Frontal lobe white matter volume was estimated using inversion recovery MRI (magnetic resonance imaging) images. A brief neuropsychological battery that focused on reaction times was performed at the end of the study. White matter volume remained stable in the LAI risperidone group and decreased significantly in the oral risperidone group resulting in a significant differential treatment effect, while the healthy controls had a white matter change intermediate and not significantly different from the two schizophrenia groups. White matter increase was associated with faster reaction times in tests involving frontal lobe function. The results suggest that LAI risperidone may improve the trajectory of myelination in first-episode patients and has a beneficial 
impact on cognitive performance. Better adherence provided by LAI AP2G may underlie the modified trajectory of myelin development [45].

\section{Benefits of Long-Acting Injectable Antipsychotics in First-Episode Patients with Schizophrenia: Clinical Perspective and Summary}

LAI AP2G have been used in clinical practice for several years. Nowadays, LAI AP2G are reserved mainly for patients with long-term course of schizophrenia who show low adherence to oral medication. The studies performed especially with LAI risperidone in patients with first episode or early stage of schizophrenia clearly indicated that this form of therapy could be effective and well tolerated also in this subgroup of patients with schizophrenia. Thanks to the familiar mother molecule (paliperidone is $9-\mathrm{OH}$ risperidone, which is the active metabolite of risperidone) data concerning efficacy and tolerability of LAI risperidone in first-episode schizophrenia, patients could be applicable for paliperidone palmitate as well. Combined benefits of AP2G characteristics with an assured route of administration raise a question whether LAI AP2G should be recommended also in first-episode patients [46, 47]. Instead of foreknowing/guessing whether patients will accept LAI therapy or not, psychiatrists should offer this form of treatment as a routine choice to all appropriate patients with schizophrenia, including first-episode subjects. Selection between long-term injectable therapy and oral medication should be based on educational and therapeutic dialogue of the psychiatrist and the patient who can then in turn discuss the potential benefits and disadvantages of the proposed therapeutic strategy $[48,49]$.

Approximately $80 \%$ of patients with a first episode of schizophrenia reach symptomatic remission after antipsychotic therapy. However, within two years most of them relapse mainly due to low insight into the illness and non-adherence to oral medication. Therefore, although the formal data available are limited many experts recommend prescribing long-acting second-generation antipsychotics (especially LAI risperidone or alternatively paliperidone palmitate) in the early stages of schizophrenia, particularly in patients who benefited from the original oral molecule in the past and agree to receive long-term injection treatment. Early application of long-acting injectable second-generation antipsychotics can significantly reduce the risk of relapses in future and thus improve not only social and working potential of patients with schizophrenia but also their quality of life.

\section{Acknowledgment}

This work was supported by the Research Plan MSM0021622404 of the Ministry of Education, Youth and Sports of the Czech Republic.

\section{References}

[1] J. M. Davis, L. Metalon, M. D. Watanabe, and L. Blake, "Depot antipsychotic drugs. Place in therapy," Drugs, vol. 47, no. 5, pp. 741-773, 1994.

[2] M. X. Patel, V. Nikolaou, and A. S. David, "Psychiatrists" attitudes to maintenance medication for patients with schizophrenia," Psychological Medicine, vol. 33, no. 1, pp. 83-89, 2003.

[3] J. Tiihonen, K. Wahlbeck, J. Lönnqvist et al., "Effectiveness of antipsychotic treatments in a nationwide cohort of patients in community care after first hospitalisation due to schizophrenia and schizoaffective disorder: observational follow-up study," British Medical Journal, vol. 333, no. 7561, pp. 224-227, 2006.

[4] P. Chue, P. M. Llorca, I. Duchesne, A. Leal, D. Rosillon, and A. Mehnert, "Hospitalization rates in patients during long-term treatment with long-acting risperidone injection," Journal of Applied Research, vol. 5, no. 2, pp. 266-274, 2005.

[5] R. Medori, R. Wapenaar, A. de Rosario et al., "Relapse prevention and effectiveness in schizophrenia with risperidone longacting injectable (RLAI) versus quetiapine," in Proceedings of the 116th Annual Convention of the APA, Boston, Mass, USA, 2008.

[6] G. M. Simpson, R. A. Mahmoud, R. A. Lasser et al., "A 1-year double-blind study of 2 doses of long-acting risperidone in stable patients with schizophrenia or schizoaffective disorder," Journal of Clinical Psychiatry, vol. 67, no. 8, pp. 1194-1203, 2006.

[7] C. Leucht, S. Heres, J. M. Kane, W. Kissling, J. M. Davis, and S. Leucht, "Oral versus depot antipsychotic drugs for schizophrenia-a critical systematic review and meta-analysis of randomised long-term trials," Schizophrenia Research, vol. 127, no. 1-3, pp. 83-92, 2011.

[8] K. Sim, A. Su, G. S. Ungvari et al., "Depot antipsychotic use in schizophrenia: an East Asian perspective," Human Psychopharmacology, vol. 19, no. 2, pp. 103-109, 2004.

[9] H. A. Nasrallah, "The case for long-acting antipsychotic agents in the post-CATIE era," Acta Psychiatrica Scandinavica, vol. 115, no. 4, pp. 260-267, 2007.

[10] J. Ahn, J. S. McCombs, C. Jung et al., "Classifying patients by antipsychotic adherence patterns using latent class analysis: characteristics of nonadherent groups in the California Medicaid (Medi-Cal) program," Value in Health, vol. 11, no. 1, pp. 48-56, 2008.

[11] S. Heres, J. Hamann, W. Kissling, and S. Leucht, "Attitudes of psychiatrists toward antipsychotic depot medication," Journal of Clinical Psychiatry, vol. 67, no. 12, pp. 1948-1953, 2006.

[12] R. W. Buchanan, J. Kreyenbuhl, D. L. Kelly et al., "The 2009 schizophrenia PORT psychopharmacological treatment recommendations and summary statements," Schizophrenia Bulletin, vol. 36, no. 1, pp. 71-93, 2010.

[13] J. Raboch, M. Anders, P. Hellerová, and P. Uhlíková, "Psychiatrie: doporučené postupy psychiatrické péče III," Tribun EU, pp. 118-126, 2010.

[14] National Institute for Health and Clinical Excellence (NICE), "Schizophrenia: Core Interventions in the Treatment and Management of Schizophrenia in Adults in Primary and Secondary Care (Update), Final Version (NICE Clinical Guideline 82). NICE, London, UK," 2009, http://www.nice.org. uk/nicemedia/pdf/CG82NICEGuideline.pdf. 
[15] D. Robinson, M. G. Woerner, J. M. J. Alvir et al., "Predictors of relapse following response from a first episode of schizophrenia or schizoaffective disorder," Archives of General Psychiatry, vol. 56, no. 3, pp. 241-247, 1999.

[16] J. A. Lieberman, T. Scott Stroup, J. P. McEvoy et al., "Effectiveness of antipsychotic drugs in patients with chronic schizophrenia," New England Journal of Medicine, vol. 353, no. 12, pp. 1209-1223, 2005.

[17] R. S. Kahn, W. W. Fleischhacker, H. Boter et al., "Effectiveness of antipsychotic drugs in first-episode schizophrenia and schizophreniform disorder: an open randomised clinical trial," The Lancet, vol. 371, no. 9618, pp. 1085-1097, 2008.

[18] P. M. Haddad and S. G. Sharma, "Adverse effects of atypical antipsychotics: differential risk and clinical implications," CNS Drugs, vol. 21, no. 11, pp. 911-936, 2007.

[19] J. Kreyenbuhl, E. P. Slade, D. R. Medoff et al., "Time to discontinuation of first- and second-generation antipsychotic medications in the treatment of schizophrenia," Schizophrenia Research, vol. 131, no. 1-3, pp. 127-132, 2011.

[20] J. A. Cramer and R. Rosenheck, "Compliance with medication regimens for mental and physical disorders," Psychiatric Services, vol. 49, no. 2, pp. 196-201, 1998.

[21] J. P. Lacro, L. B. Dunn, C. R. Dolder, S. G. Leckband, and D. V. Jeste, "Prevalence of and risk factors for medication nonadherence in patients with schizophrenia: a comprehensive review of recent literature," Journal of Clinical Psychiatry, vol. 63, no. 10, pp. 892-909, 2002.

[22] P. Thieda, S. Beard, A. Richter, and J. Kane, "An economic review of compliance with medication therapy in the treatment of schizophrenia," Psychiatric Services, vol. 54, no. 4, pp. 508-516, 2003.

[23] J. L. Young, H. V. Zonana, and L. Shepler, "Medication noncompliance in schizophrenia: codification and update," Bulletin of the American Academy of Psychiatry and the Law, vol. 14, no. 2, pp. 105-122, 1986.

[24] J. L. Young, R. T. Spitz, M. Hillbrand, and G. Daneri, "Medication adherence failure in schizophrenia: a forensic review of rates, reasons, treatments, and prospects," Journal of the American Academy of Psychiatry and the Law, vol. 27, no. 3, pp. 426-444, 1999.

[25] L. Shi, H. Ascher-Svanum, B. Zhu, D. Faries, W. Montgomery, and S. R. Marder, "Characteristics and use patterns of patients taking first-generation depot antipsychotics or oral antipsychotics for schizophrenia," Psychiatric Services, vol. 58, no. 4, pp. 482-488, 2007.

[26] J. Tiihonen, J. Haukka, M. Taylor, P. M. Haddad, M. X. Patel, and P. Korhonen, "A nationwide cohort study of oral and depot antipsychotics after first hospitalization for schizophrenia," American Journal of Psychiatry, vol. 168, no. 6, pp. 603609, 2011.

[27] C. E. Adams, M. K. P. Fenton, S. Quraishi, and A. S. David, "Systematic meta-review of depot antipsychotic drugs for people with schizophrenia," British Journal of Psychiatry, vol. 179, pp. 290-299, 2001.

[28] P. M. Haddad, M. Taylor, and O. S. Niaz, "First-generation antipsychotic long-acting injections v. oral antipsychotics in schizophrenia: systematic review of randomised controlled trials and observational studies," British Journal of Psychiatry, vol. 195, no. 52, supplement, pp. s20-s28, 2009.

[29] E. L. Coldham, J. Addington, and D. Addington, "Medication adherence of individuals with a first episode of psychosis," Acta Psychiatrica Scandinavica, vol. 106, no. 4, pp. 286-290, 2002.

[30] R. Emsley, P. Oosthuizen, L. Koen et al., "A study of the clinical outcome following treatment discontinuation after remission in first-episode schizophrenia," European Neuropsychopharmacology, vol. 19, no. 3, supplement, pp. 486-487, 2009.

[31] L. Waddell and M. Taylor, "Attitudes of patients and mental health staff to antipsychotic long-acting injections: systematic review," British Journal of Psychiatry, vol. 195, no. 52, pp. s43s50, 2009.

[32] M. X. Patel, P. M. Haddad, I. B. Chaudhry, S. McLoughlin, and A. S. David, "Psychiatrists' use, knowledge and attitudes to first- and second-generation antipsychotic long-acting injections: comparisons over 5 years," Journal of Psychopharmacology, vol. 24, no. 10, pp. 1473-1482, 2010.

[33] S. Heres, T. Reichhart, J. Hamann, R. Mendel, S. Leucht, and W. Kissling, "Psychiatrists' attitude to antipsychotic depot treatment in patients with first-episode schizophrenia," European Psychiatry, vol. 26, pp. 297-301, 2011.

[34] A. F. Lehman, J. A. Lieberman, L. B. Dixon et al., "Practice guideline for the treatment of partients with schizophrenia, 2nd Edition," American Journal of Psychiatry, vol. 161, no. 2, pp. 1-56, 2004.

[35] P. Falkai, T. Wobrock, J. Lieberman et al., "World Federation of Societies of Biological Psychiatry (WFSBP) guidelines for biological treatment of schizophrenia-part 2: longterm treatment of schizophrenia," World Journal of Biological Psychiatry, vol. 7, no. 1, pp. 5-40, 2006.

[36] C. Mentschel, "Depot-drugs may reduce relapse rates in schizophrenic outpatients: a meta-analysis," in Proceedings of the 156th meeting of the American Psychiatric Association, p. 71, San Francisco, Calif, USA, 2003.

[37] P. J. Weiden, N. R. Schooler, J. C. Weedon, A. Elmouchtari, A. Sunakawa, and S. M. Goldfinger, "A randomized controlled trial of long-acting injectable risperidone vs continuation on oral atypical antipsychotics for first-episode schizophrenia patients: initial adherence outcome," Journal of Clinical Psychiatry, vol. 70, no. 10, pp. 1397-1406, 2009.

[38] M. X. Patel, M. Taylor, and A. S. David, "Antipsychotic longacting injections: mind the gap," British Journal of Psychiatry, vol. 195, no. 52, supplement, pp. s1-s4, 2009.

[39] E. Parellada, R. Andrezina, V. Milanova et al., "Patients in the early phases of schizophrenia and schizoaffective disorders effectively treated with risperidone long-acting injectable," Journal of Psychopharmacology, vol. 19, no. 5, pp. 5-14, 2005.

[40] H. J. Möller, P. M. Llorca, E. Sacchetti, S. D. Martin, R. Medori, and E. Parellada, "Efficacy and safety of direct transition to risperidone long-acting injectable in patients treated with various antipsychotic therapies," International Clinical Psychopharmacology, vol. 20, no. 3, pp. 121-130, 2005.

[41] S. R. Kay, A. Fiszbein, and L. A. Opler, "The positive and negative syndrome scale (PANSS) for schizophrenia," Schizophrenia Bulletin, vol. 13, no. 2, pp. 261-276, 1987.

[42] N. C. Andreasen, W. T. Carpenter, J. M. Kane, R. A. Lasser, S. R. Marder, and D. R. Weinberger, "Remission in schizophrenia: proposed criteria and rationale for consensus," American Journal of Psychiatry, vol. 162, no. 3, pp. 441-449, 2005.

[43] R. Emsley, P. Oosthuizen, L. Koen, D. J. H. Niehaus, R. Medori, and J. Rabinowitz, "Remission in patients with first-episode schizophrenia receiving assured antipsychotic medication: a study with risperidone long-acting injection," International Clinical Psychopharmacology, vol. 23, no. 6, pp. 325-331, 2008.

[44] A. Malla, C. Binder, and P. Chue, "Comparison of longacting injectable risperidone and oral novel antipsychotic drugs for treatment in early phase of schizophrenia spectrum psychosis," in Proceedings of the 61st Annual Convention Society of Biological Psychiatry, Toronto, Canada, 2006. 
[45] G. Bartzokis, P. H. Lu, C. P. Amar et al., "Long acting injection versus oral risperidone in first-episode schizophrenia: differential impact on white matter myelination trajectory," Schizophrenia Research, vol. 132, no. 1, pp. 35-41, 2011.

[46] P. Chue and R. Emsley, "Long-acting formulations of atypical antipsychotics: time to reconsider when to introduce depot antipsychotics," CNS Drugs, vol. 21, no. 6, pp. 441-448, 2007.

[47] M. X. Patel and A. S. David, "Why aren't depot antipsychotics prescribed more often and what can be done about it?" Advances in Psychiatric Treatment, vol. 11, no. 3, pp. 203-213, 2005.

[48] T. Lambert, "Selecting patients for long-acting novel antipsychotic therapy," Australasian Psychiatry, vol. 14, no. 1, pp. 3842, 2006.

[49] R. Fraser, G. Berger, E. Killackey, and P. McGorry, "Emerging psychosis in young people-part 3- key issues for prolonged recovery," Australian Family Physician, vol. 35, no. 5, pp. 329333, 2006. 


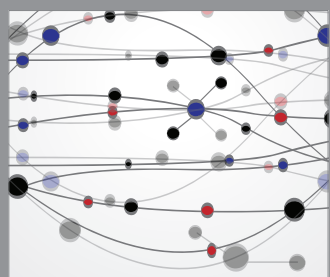

The Scientific World Journal
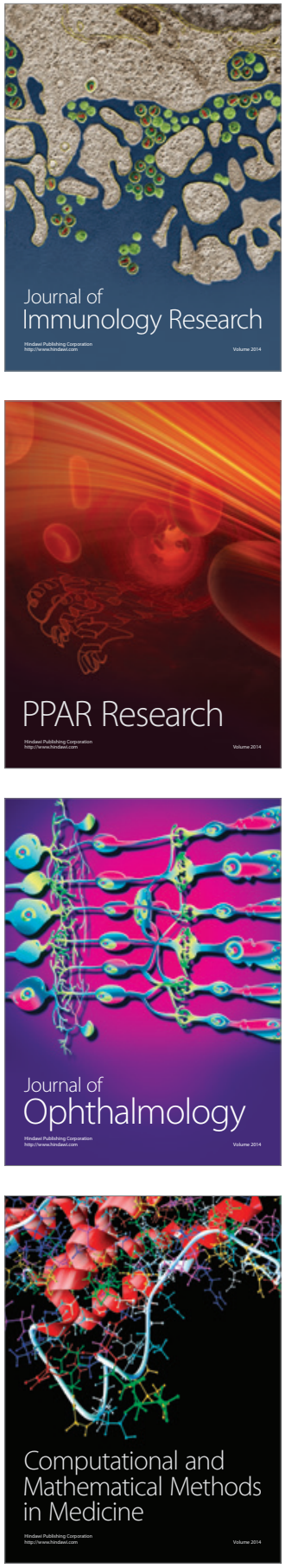

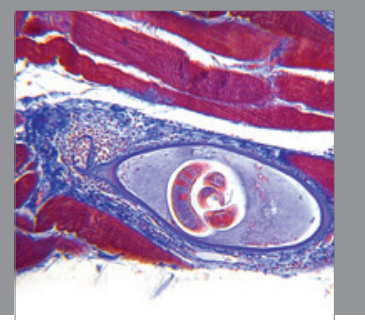

Gastroenterology

Research and Practice
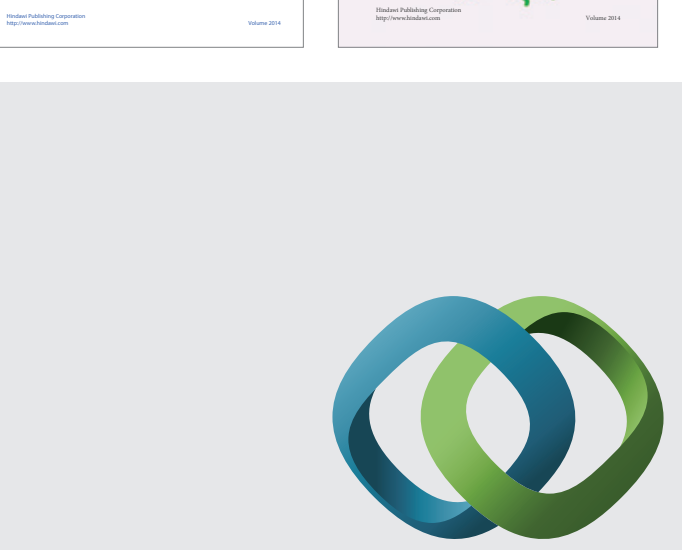

\section{Hindawi}

Submit your manuscripts at

http://www.hindawi.com
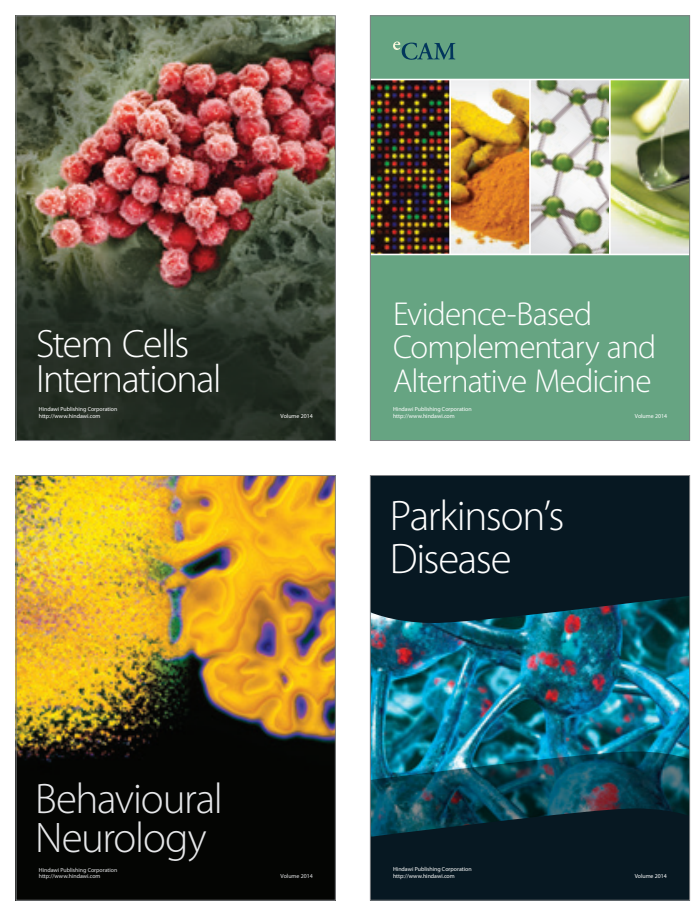

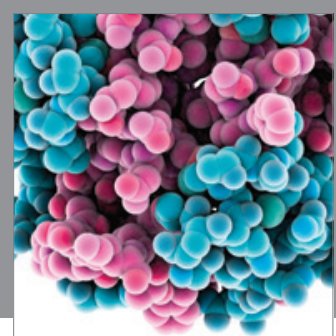

Journal of
Diabetes Research

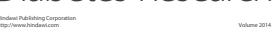

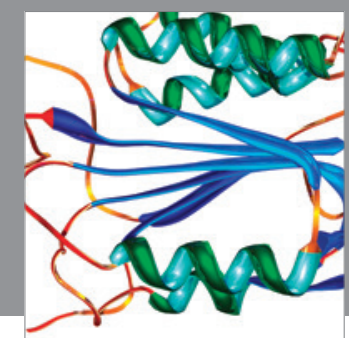

Disease Markers
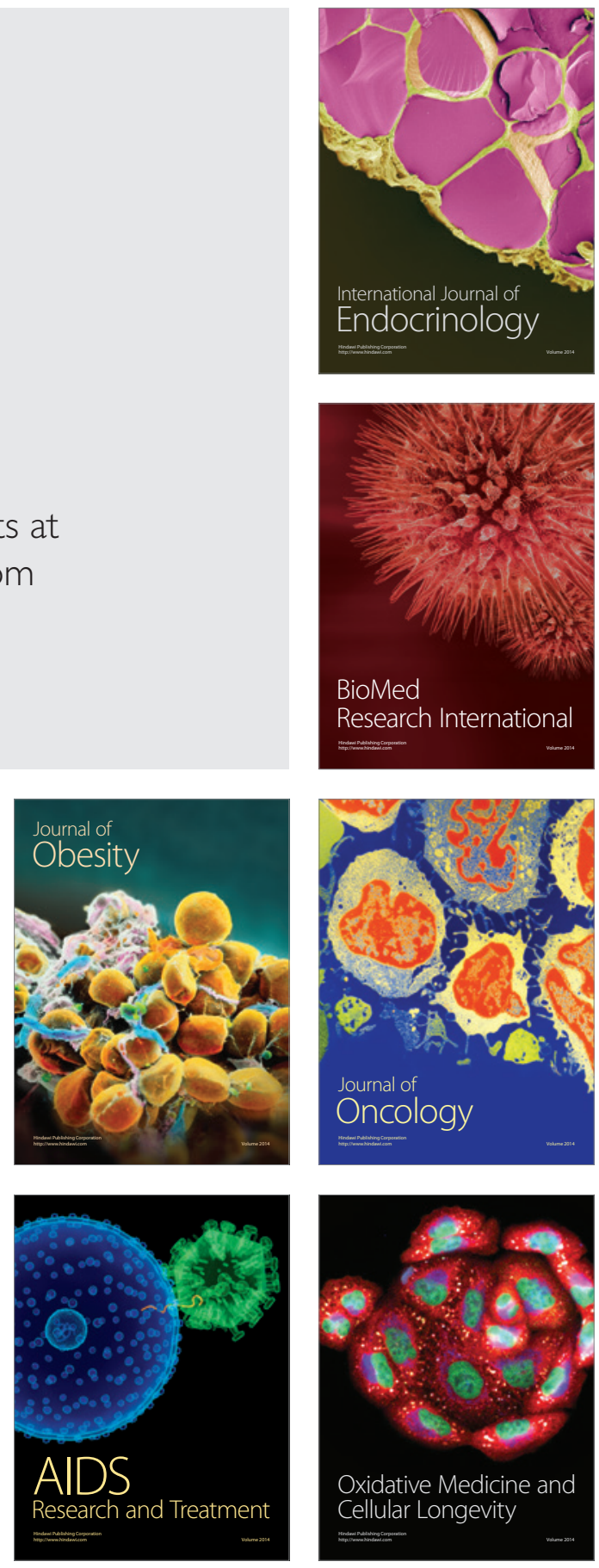